\title{
TUNNELING IN CUPRATE AND BISMUTHATE SUPERCONDUCTORS*
}

\author{
J.F. Zasadzinski, Qiang Huang, N. Tralshawala \\ Department of Physics \\ Illinois Institute of Technology, Chicago, IL 60616 \\ and \\ K.E. Gray \\ Materials Science Division \\ Argonne National Laboratory, Argonne, Illinois 60439
}

DEC 131991

Fifth Annual Conference on Superconductivity and Applications, Buffalo, NY, September 24-26, 1991

jmc

\section{DISCLAIMER}

\begin{abstract}
This report was prepared as an account of work sponsored by an agency of the United States Government. Neither the United States Government nor any agency thereof, nor any of their employees, makes any warranty, express or implied, or assumes any legal liability or responsibility for the accuracy, completeness, or usefulness of any information, apparatus, product, or process disclosed, or represents that its use would not infringe privately owned rights. Reference herein to any specific commercial product, process, or service by trade name, trademark, manufacturer, or otherwise does not necessarily constitute or imply its endorsement, recommendation, or favoring by the United States Government or any agency thereof. The views and opinions of authors expressed herein do not necessarily state or reflect those of the United States Government or any agency thereof.
\end{abstract}

\footnotetext{
"Work supported by the National Science Foundation-Office of Science and Technology Centers for Superconductivity under contract \#DMR-8809854 (JFZ, QH) and the U.S. Department of Energy, Division of Basic Energy Sciences-Materials Sciences under contract \#W-31-109-ENG-38. 


\section{TUNNELING IN CUPRATE AND BISMUTHATE SUPERCONDUCTORS}

J. F. Zasadzinski*, Q. Huang and N. Tralshawala

Illinois Institute of Technology, Chicago, IL. 60616

K. E. Gray

Materials Science Division,

Argonne National Laboratory, Argonne, IL. 60439

\section{ABSTRACT}

Tunneling measurements using a point-contact technique are reported for the following high temperature superconducting oxides: $\mathrm{Ba}_{1-\mathrm{x}} \mathrm{K}_{\mathrm{x}} \mathrm{BiO}_{3}(\mathrm{BKBO}), \mathrm{Nd}_{2}-\mathrm{x} \mathrm{Ce}_{\mathrm{x}} \mathrm{CuO}_{4}$ (NCCO), $\mathrm{Bi}_{2} \mathrm{Sr}_{2} \mathrm{CaCu}_{2} \mathrm{O}_{7}$ (BSCCO) and $\mathrm{Tl}_{2} \mathrm{Ba}_{2} \mathrm{CaCu}_{2} \mathrm{O}_{\mathrm{x}}$ (TBCCO). For the bismuthate, $\mathrm{BKBO}$, ideal, S-I-N tunneling characteristics are observed using a $\mathrm{Au}$ tip. The normalized conductance is fitted to a BCS density of states and thermal smearing only proving there is no fundamental limitation in BKBO for device applications. For the cuprates, the normalized conductance displays BCS-like characteristics, but with a broadening larger than from thermal smearing. Energy gap values are presented for each material. For BKBO and NCCO the Eliashberg functions, $\alpha^{2} F(\omega)$, obtained from the tunneling are shown to be is good agreement with neutron scattering results. Proximity effect tunneling studies are reported for $\mathrm{Au} / \mathrm{BSCCO}$ bilayers and show that the energy gap of BSCCO can be observed through Au layers up to $600 \AA$ thick.

\section{INTRODUCTION}

Tunneling spectroscopy is a very powerful probe of the superconducting state ${ }^{1}$ and, in ideal cases, provides a direct measure of the energy dependent gap function, $\Delta(\omega)$, and the electron-phonon spectral function, $\alpha^{2} F(\omega)$. In addition, a number of applications exist for thin-film tunnel junctions, including photon detectors, frequency standards and fast switches. Thus it is not surprising that a world-wide effort exists to form high quality

*Work supported by the National Science Foundation-Office of Science and Technology Centers (DMR 88-09854) and U.S. Department of Energy, Division of Basic Energy Sciences-Materials Sciences under contract \#W-31-109-ENG-38. 
junctions on high temperature superconductors (HTS) for fundamental studies and technological purposes. Unfortunately, the tunnel junctions obtained on cuprate superconductors have been far from ideal, showing BCS-like features, but with varying degrees of broadening and evidence of quasiparticle states inside the gap. In fact, the question of whether there exists a true energy gap in the cuprates remains an open one. Thus far, the bismuthate superconductor, $\mathrm{Ba}_{1-\mathrm{x}} \mathrm{K}_{\mathrm{x}} \mathrm{BiO}_{3}(\mathrm{BKBO})$, is the only oxide with a $\mathrm{Tc}>25 \mathrm{~K}$ to display a well-defined energy gap 2 in a tunneling measurement.

Inroads into the probiem of tunneling in HTS have been made by using a point-contact method, where the tip can be used to scrape, clean and even cleave the sample surface. We report here some of our best results on cuprate and bismuthate superconductors using this technique. Ideal junctions are found on $\mathrm{BKBO}$ with the normalized tunneling conductance fitted very well to a thermally smeared BCS density of states (dos). For $\mathrm{Bi}_{2} \mathrm{Sr}_{2} \mathrm{CaCu}_{2} \mathrm{O}_{7}(\mathrm{BSCCO})^{3}$ and $\mathrm{Nd}_{2}-\mathrm{Ce}_{2} \mathrm{CuO}_{4}(\mathrm{NCCO})^{4}$ we have observed a zero bias dos as low as $10 \%$, which is a significant improvement over previously reported data. Structures have been found ${ }^{4}$ in the high bias data of BKBO and NCCO which have the characteristics of phonon effects as found in conventional superconductors. We show that the $\alpha^{2} \mathrm{~F}(\omega)$ for $\mathrm{BKBO}$ and $\mathrm{NCCO}$ bear a close resemblance to the phonon density of states measured by neutron scattering, indicating that electron pairing is princir ally mediated by phonons.

To address the problem of broadening of the tunneling dos in cuprates, we have investigated a proximity effect approach whereby a thin layer of $\mathrm{Au}(200-600 \AA)$ is deposited onto a freshly cleaved BSCCO crystal. The results show that the BSCCO energy gap is observed throught the Au layer, and for a $200 \AA$ layer, the zero bias dos appears to be close to zero. This approach may thus be suitable to fabricate thin film juntions with low subgap currents and sharp onsets of current at the gap voltage, characteristics which are essential for device purposes.

\section{ENERGY GAP MEASUREMENTS}

In an ideal tunneling experiment, the dynamic conductance, $\sigma=\mathrm{dI} / \mathrm{dV}$, measured in the superconducting and normal state, leads directly to the quasiparticle $\operatorname{dos}^{1}, N(E)$, by the expression

$$
N(E)=\sigma_{s} / \sigma_{n}=\operatorname{Re} E^{2} /\left(E^{2}-\Delta^{2}(E)\right)^{1 / 2} \quad \text { Eq. } 1
$$


Here, $\Delta(E)$ is the complex gap parameter which, in conventional superconductors, has a strong energy dependence near energies which correspond to peaks in the phonon dos owing to the inelastic scattering of electrons by phonons. However for lower energies, $\Delta(E)$ is approximately a real constant, $\Delta$, and Eq. 1 becomes the standard BCS dos. Finite temperature effects smear out $\mathrm{N}(\mathrm{E})$ by approximately $3.5 \mathrm{kT}$. An example of an ideal result is shown in Fig. 1 for a point-contact tunnel junction on the cubic bismuthate, $\mathrm{BKBO}(\mathrm{Tc}=25-30 \mathrm{~K})$ at $4.2 \mathrm{~K}$ using a Au tip.

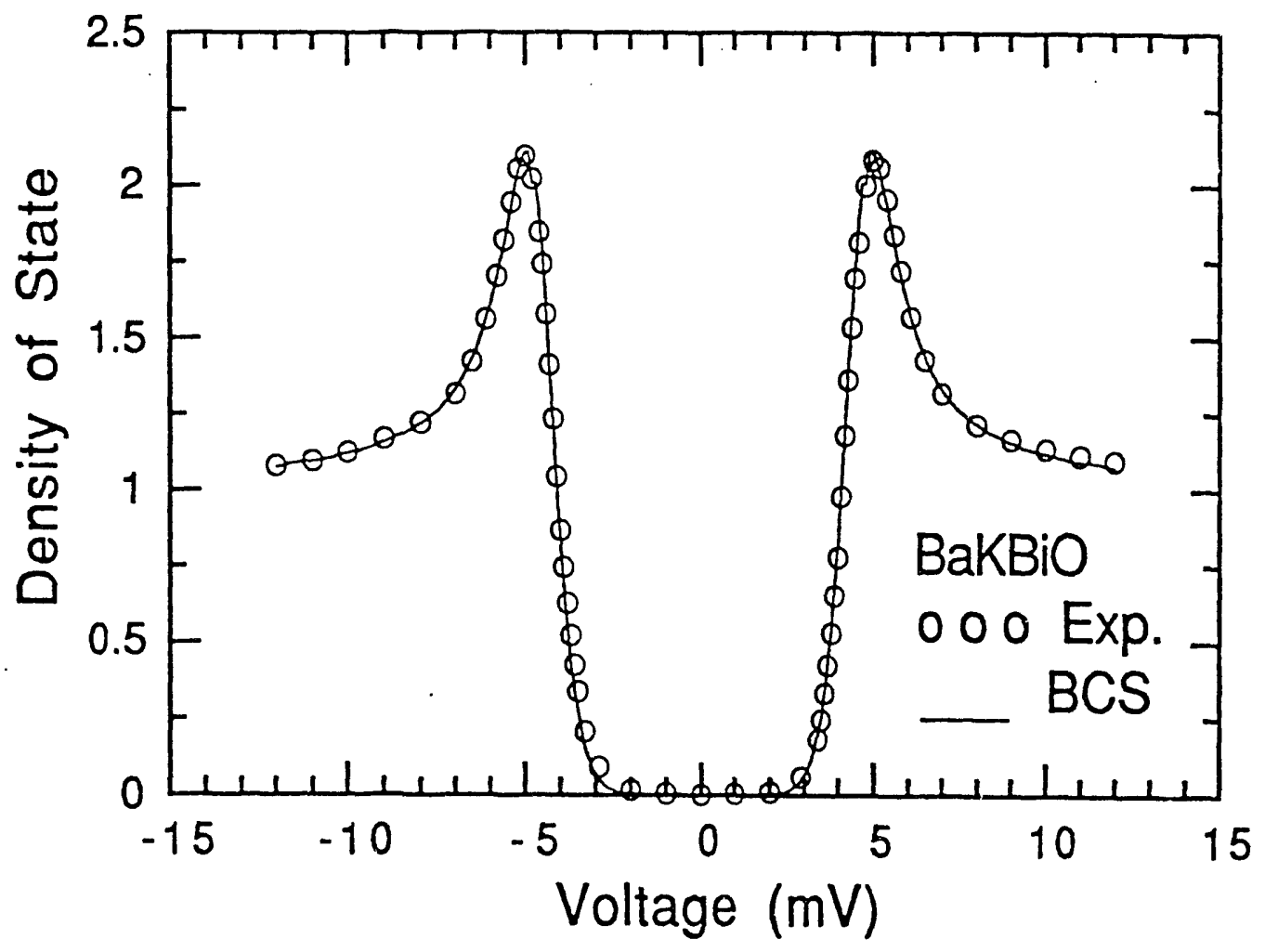

Figure 1. Fit of the experimental normalized conductance, open circles, with a BCS dos and thermal smearing of $4.2 \mathrm{~K}$ (solid line). The gap parameter, $\Delta=4.5 \mathrm{meV}$.

The experimental data are fit quite well to the BCS dos with a single value of $\Delta=4.5 \mathrm{meV}$ and thermal smearing only. Note the flat, near-zero dos for voltages, $\mathrm{eV}<\Delta$, which proves that $\mathrm{BKBO}$ has a well-defined energy gap. Moving the Au tip from point to point on the polycrystalline sample, data similar to Fig. 1 were obtained although the value of $\Delta$ ranged from $3.6-4.6 \mathrm{meV}$. The different $\Delta$ values are likely due to variations of $\mathrm{K}$ concentration from grain to grain and associating the large(small) $\Delta$ values with the $10 \%(90 \%)$ points of the magnetic transition of this sample, we obtain $2 \Delta / \mathrm{kT} c$ $=3.8-3.9$. This indicates moderate coupling strength. Using a $\mathrm{Nb}$ 
tip, very low sub-gap conductance is found $(<0.2 \%)^{2}$ as well as a sharp current onset at the sum gap, proving there is no fundamental limitation in $\mathrm{BKBO}$ for device applications.

The electron-doped superconductor, NCCO, has a relatively low $\mathrm{Tc} \sim 23 \mathrm{~K}$, but shares many of the properties of the higher $\mathrm{T}_{\mathrm{C}}$ cuprates, including the quasi two-dimensional $\mathrm{Cu}-\mathrm{O}$ planes. Generally high quality tunneling results were consistently obtained on single crystals of NCCO and a representative normalized conductance is shown in Fig. 2.

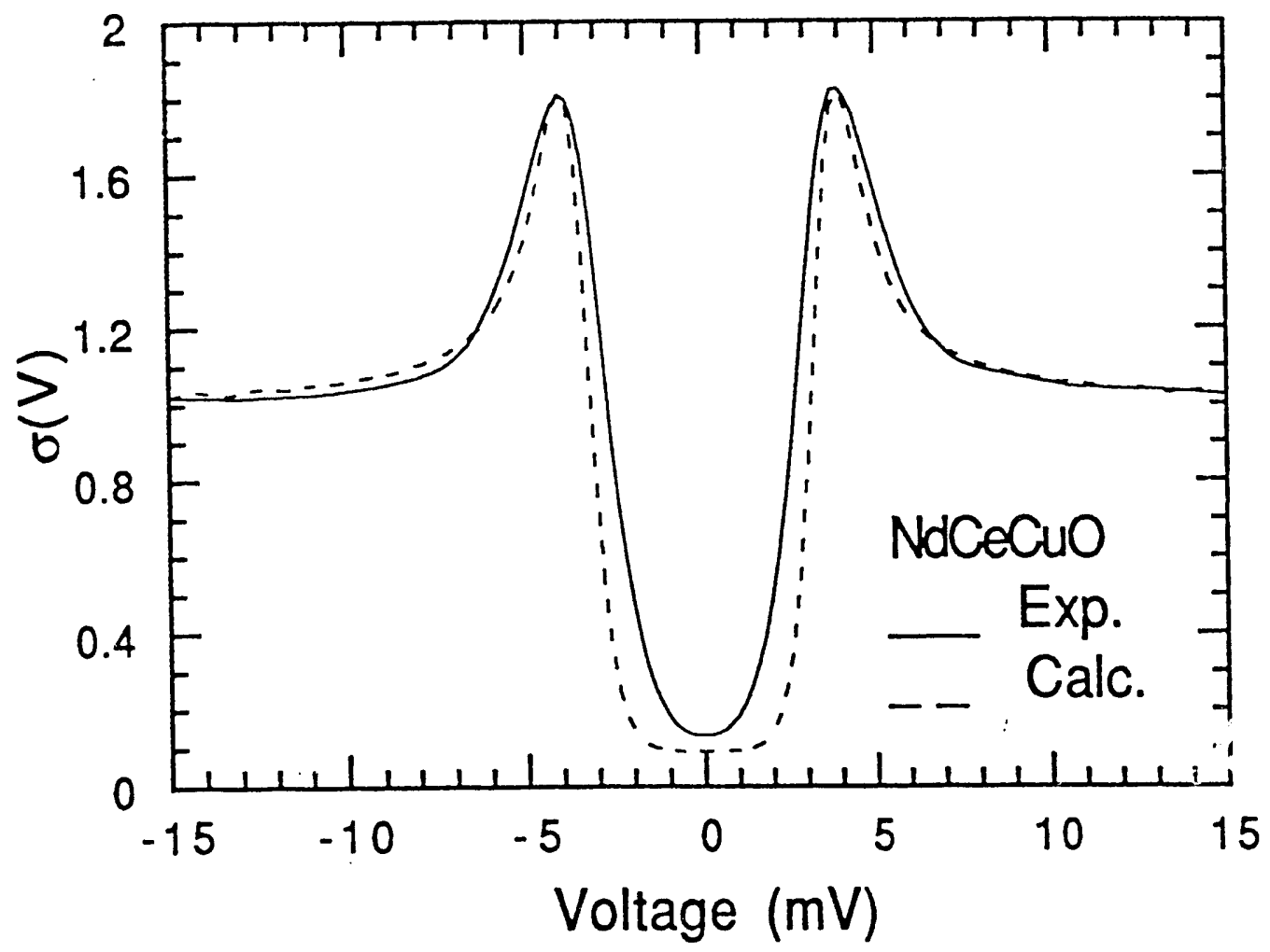

Figure 2. Experimental normalized tunneling conductance (solid line) for NCCO. Thermally smeared BCS dos (dashed line) includes a leakage conductance of $10 \%$. The gap parameter, $\Delta=3.6 \mathrm{meV}$.

While the data diplay clear evidence of the BCS dos, there appears to be smearing of the structure in addition to that due to temperature effects and the normalized conductance at zero bias is not zero but $10-15 \%$. However, the structure is much sharper than found in earlier studies. $5 \mathrm{We}$ have fit the data with a thermally smeared BCS dos including a constant leakage conductance of $10 \%$ and while the fit is reasonably good, there are obvious differences in the two curves. Most notably, the shape of the data near zero-bias has a parabolic character while the BCS dos has the expected flat behavior indicative of a true energy gap. 
The shape of the data indicates that the zero-bias dos is probably not due to leakage at all, but rather is manifestation of the overall broadening.

Despite the non-ideal fit of the NCCO data, the structures are sharp enough to extract the gap parameter, and a value $\Delta=3.5-3.6$ meV was consistently obtained for over 15 junctions. Using the width of the magnetic transition, we obtain $2 \Delta / \mathrm{kT}_{\mathrm{c}}=3.5-4.1$, indicating weak to moderate coupling strength.

Tunneling data on higher $\mathrm{T}_{\mathrm{c}}$ cuprates such as $\mathrm{BSCCO}^{3,6}$ $\left(\mathrm{T}_{\mathrm{C}}=85 \mathrm{~K}\right)$ and $\mathrm{TBCCO}^{7,8}\left(\mathrm{~T}_{\mathrm{C}}=120 \mathrm{~K}\right)$ generally display much more broadening than we observe in NCCO. An example of one of our best tunneling curves for $\mathrm{TBCCO}$ is shown in Fig. 3. Important features to note include the reduced size of the normalized conductance peaks (1.4 compared to 2.1 in $\mathrm{BKBO}$ ) and the zerobias value of $\sim 25 \%$. These features are evidence of increased broadening of the BCS dos and thus we have attempted to fit the data using a phenomenological expression which adds an imaginary part, $\Gamma$, to the energy, $E$, in eq. 1 . The value of $I$ is thus a measure of the degree of broadening.

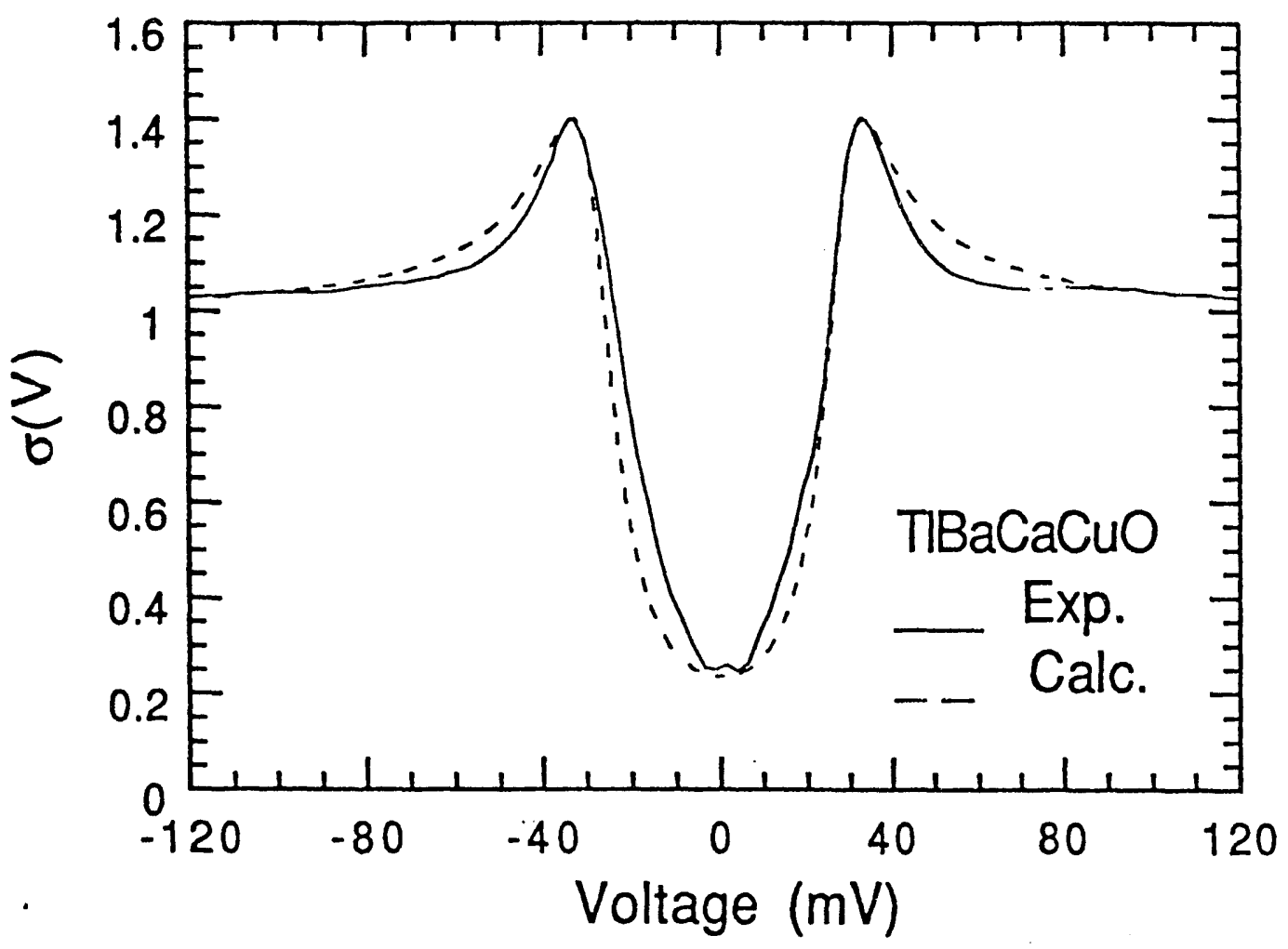

Figure 3. Experimental normalized conductance for TBCCO (solid line). The fit (dashed line) is a broadened BCS dos as explained in the text with $\Delta=28 \mathrm{meV}$ and $\Gamma=6.8 \mathrm{meV}$. 
The fit of the TBCCO data is reasonably good, showing how the broadening of the BCS dos leads to reduced conductance peaks and a non-zero value of the zero-bias conductance. Another important feature is that the gap parameter for the fit in Fig. 3 $(\Delta=28 \mathrm{meV})$ is significantly less than the voltage of the conductance peaks $(\mathrm{eV}= \pm 32 \mathrm{meV})$, and demonstrates that the extraction of the gap parameter directly from this voltage leads to an overestimate of the gap. It should be noted that for the cuprates with $\mathrm{T}_{\mathrm{C}}>77 \mathrm{~K}$, a direct measurement of the normal state curve is very difficult using the point contact method. Thus we have generated the normal state data from high-bias superconducting data using a procedure described elsewhere. 3,8

Another important HTS material is BSCCO and the general results for the normalized tunneling conductance in this compound are similar to that obtained for TBCCO, namely, broadened BCS-like features with zero-bias values in the range $20-50 \%$. We have steadily improved the sharpness of the gap region tunneling data in $\mathrm{BSCCO}^{3}$ using the point-contact method and one of our best results to date is shown in Fig. 4.

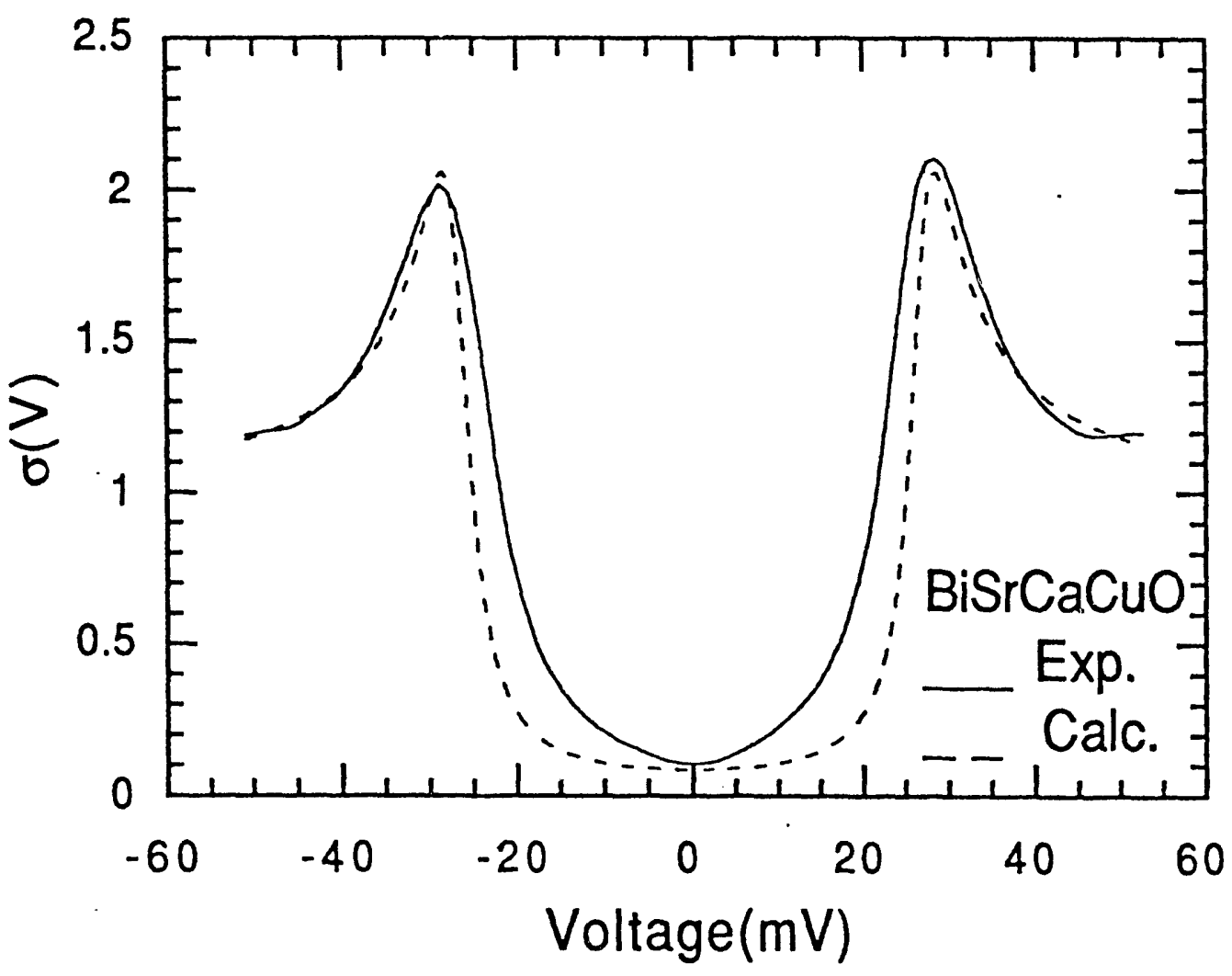

Figure 4. Experimental normalized conductance (solid line) for BSCCO compared to broadened BCS dos (dashed line) with parameters, $\Delta=27 \mathrm{meV}$, and $\Gamma=2.3 \mathrm{meV}$. 
What is most notable about the BSCCO data in Fig. 4 is the similar shape to the NCCO data of Fig. 2 for absolute voltages $e V<\Delta$. It appears that for those junctions which exhibit relatively sharp structure (zero bias values 10-15\%), the normalized conductance has a parabolic-like shape near zero bias whereas the broadened $\mathrm{BCS}$ dos is flat. This indicates that neither thermal smearing plus leakage nor the phenomenological broadening introduced with $\Gamma$ accurately describes the experimental data. Nevertheless, the gap parameters obtained with such fitting procedures are expected to be more accurate than obtained with constructs such as using the voltage of the conductance peak. The gap parameters and $2 \Delta / \mathrm{kT}_{\mathrm{c}}$ values obtained from all the data sets are summarized in Table 1.

Table 1. Experimental Gap Parameters

\begin{tabular}{l|ccc}
\hline \hline Material & $\Delta(\mathrm{meV})$ & $\mathrm{T}_{\mathrm{c}}(\mathrm{K})$ & $2 \Delta / \mathrm{k}_{\mathrm{B}} \mathrm{T}_{\mathrm{c}}$ \\
\hline $\mathrm{Ba}_{1-\mathrm{x}} \mathrm{K}_{\mathrm{x}} \mathrm{BiO}_{3}$ & $3.6 \sim 4.6$ & $21.5 \sim 27.5$ & $3.8 \sim 3.9$ \\
$\mathrm{Nd}_{2-\mathrm{x}} \mathrm{Ce}_{\mathrm{x}} \mathrm{CuO}_{4-\mathrm{y}}$ & $3.5 \sim 3.7$ & $20 \sim 24$ & $3.5 \sim 4.1$ \\
$\mathrm{Bi}_{2} \mathrm{Sr}_{2} \mathrm{CaCu}_{2} \mathrm{O}_{\mathrm{x}}$ & $16 \sim 30$ & $86 \sim 96$ & $4.3 \sim 7.3$ \\
$\mathrm{Tl}_{2} \mathrm{Ba}_{2} \mathrm{CaCu}_{2} \mathrm{O}_{\mathrm{x}}$ & $16 \sim 28$ & $90 \sim 112$ & $4.1 \sim 5.8$ \\
\hline
\end{tabular}

\section{PHONON SPECTROSCOPY}

The high quality tunneling data observed for $\mathrm{BKBO}$ and NCCO coupled with the ability to measure the normal state conductance in these relatively low Tc materials, makes tunneling spectroscopy a possibility. As mentioned in the Introduction, the high bias tunneling data for conventional superconductors such as $\mathrm{Pb}$ or $\mathrm{Nb}$, deviate from the BCS dos by a few percent or less at energies which correspond to peaks in the phonon dos. We have shown that the point contact technique reproduces the phonon structures in $\mathrm{Nb}^{9}$ observed with thin-film junctions. We have observed structures in the high-bias, normalized tunneling conductance data of BKBO and NCCO which are characteristic of phonon effects as seen in conventional superconductors. Using a modified version of the McMillan-Rowell inversion program 4 which allows for a thin proximity layer on the surface of the superconductor, 
we have inverted the tunneling data to obtain the electronphonon spectral function, $\alpha^{2} F(\omega)$.

A comparison is shown in Figs. 5 and 6 between the $\alpha^{2} F(\omega)$

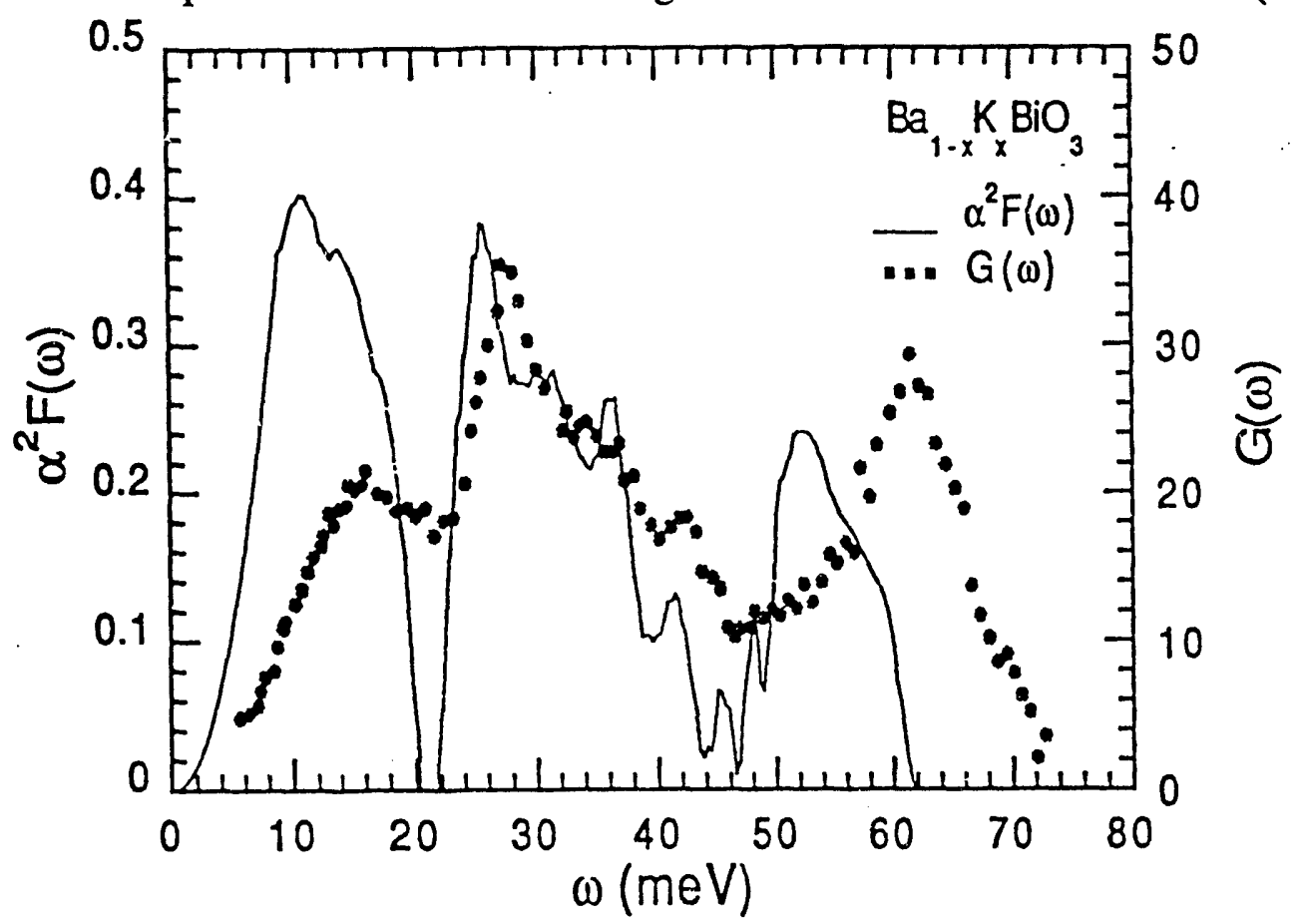

Figure 5. Comparison of $\alpha^{2} \mathrm{~F}(\omega)$ for BKBO (solid line) with the phonon $G(\omega)$ (solid circles) from neutron scattering.

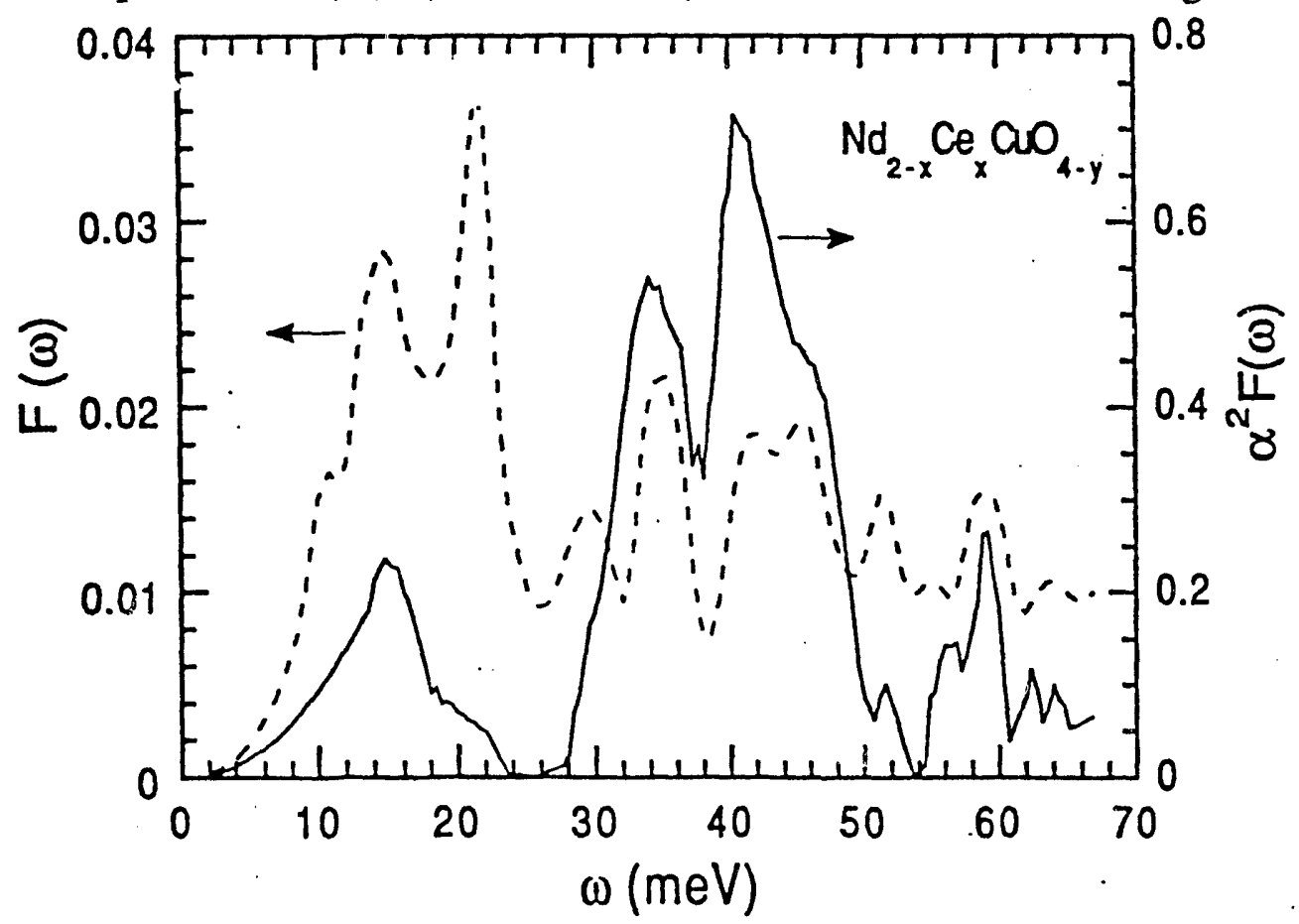

Figure 6. Comparison of $\alpha^{2} F(\omega)$ for NCCO with $F(\omega)$ (dashed line) from neutron scattering. 
for BKBO and NCCO and the phonon density of states $(F(\omega)$ or $G(\omega)$ ) obtained by neutron scattering. For BKBO the phonon $G(\omega)$ is obtained from a polycrystalline pcllet 10 and differs from $F(\omega)$ by scattering cross section terms, but the locations of peaks and valleys in the two functions should be similar. As is seen in Fig. 5, there is a good correlation of peaks and especially minima between $G(\omega)$ and $\alpha^{2} F(\omega)$ in BKBO.

For NCCO, measurements by Reichardt et al ${ }^{11}$ on single crystals have yielded dispersion curves from which the $F(\omega)$ is generated. There is a remarkable agreement on the locations of peaks and valleys between $\alpha^{2} F(\omega)$ and $F(\omega)$ although the shapes of the two curves are different suggesting that $\alpha^{2}$ has a strong energy dependence. The parameters, $\lambda$ and $\mu^{*}$, for two junctions each of BKBO and NCCO are given in Table 2, as are the calculated and measured $T_{C}$ values. As is seen there is reasonable agreement between the measured $\mathrm{Tc}$ values and those calculated from the $\alpha^{2} F(\omega)$ functions, indicating that electron pairing is mediated principally by phonons in BKBO and NCCO.

Table 2. Measured and Calculated Parameters

\begin{tabular}{|c|c|c|c|c|c|c|}
\hline Junction & $\Delta(\mathrm{meV})$ & $\lambda$ & $\mu^{*}$ & $\omega_{\log }(\mathrm{meV})$ & $\mathrm{T}_{\mathrm{c}}(\mathrm{K})$ & $\mathrm{T}_{\mathrm{c}}^{\text {calc }}(\mathrm{K})$ \\
\hline $\mathrm{BKBO} \# 1$ & $3 . i$ & $1.2 \pm 0.2$ & $0.11 \pm 0.04$ & 25.1 & $24.5 \pm 3$ & 19 \\
\hline $\mathrm{BKBO} \# 2$ & 4.5 & $1.3 \pm 0.3$ & $0.04 \pm 0.04$ & 13.7 & $24.5 \pm 3$ & 20 \\
\hline $\mathrm{NCCO} \# 1$ & 3.5 & $0.9 \pm 0.1$ & $0.05 \pm 0.05$ & 19.7 & $22.0 \pm 2$ & 21 \\
\hline $\mathrm{NCCO} \# 2$ & 3.6 & $1.0 \pm 0.1$ & $0.08 \pm 0.04$ & 25.1 & $22.0 \pm 2$ & 19 \\
\hline
\end{tabular}

\section{PROXIMITY EFFECT STUDIES}

The limitation of the point-contact technique is the difficulty in measuring the normal state conductance of a junction due to differential thermal expansion of the mechanical assembly during heating, and this is especially critical for HTS with $\mathrm{T}_{\mathrm{c}}$ values above 77K. Thin film junctions on HTS are desireable for spectroscopy and, in addition, have device potential. For these reasons, we have investigated junctions on single crystals of BSCCO which have been cleaved in vacuum and immediately coated with a thin layer of $\mathrm{Au}$ to protect the surface. An important question is whether 
the underlying energy gap of the $\mathrm{BSCCO}$ is observable through the Au layer.

In Fig. 7 is shown a selected set of tunneling data for various $\mathrm{Au}$ thicknesses in the range 200-600 $\AA$. Here a soft In tip was used so as not to perforate the Au layer and also the In-oxide surface of the tip provides a reasonably good tunnel barrier.

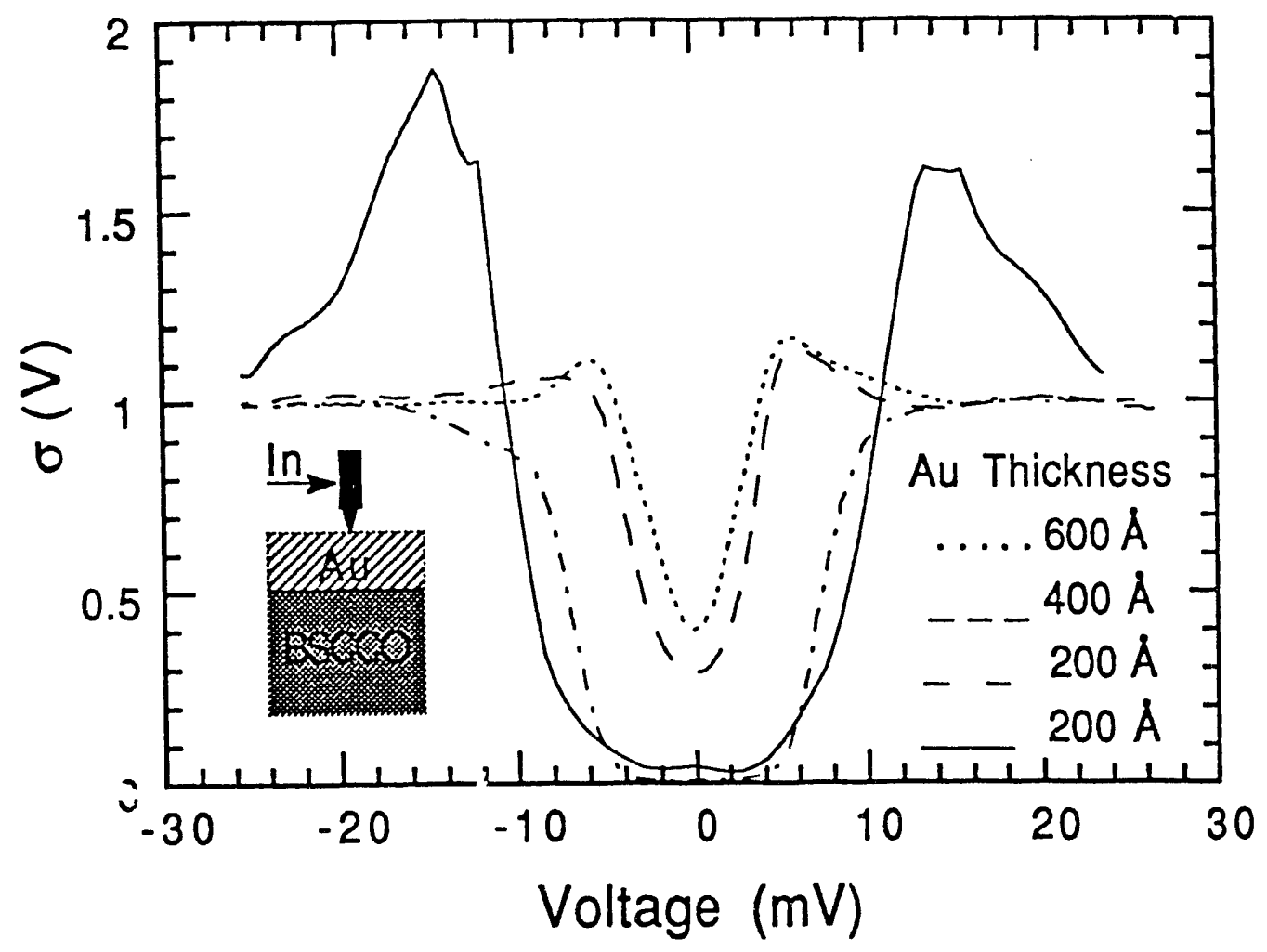

Figure 7. Normalized tunneling conductance for Au/BSCCO bilayers with various $\mathrm{Au}$ thicknesses. The tunneling geometry is shown in the inset.

The observed gap parameter of the Au/BSCCO bilayers decreases in a monotonic fashion with $\mathrm{Au}$ thickness, and even the $600 \AA$ layers typically show a gap parameter of $\sim 3 \mathrm{meV}$. This behavior can be explained using the proximity effect theory of Arnold.1 Furthermore, two junctions with Au thicknesses of $200 \AA$ showed a zero-bias dos close to zero, in contrast to point contact junctions formed directly on the BSCCO surface which always displayed at leas: $10-15 \%$ zero-bias dos. This indicates that the proximity effect approach has potential as a technique for fabricating thinfilm junctions with low sub-gap conductance which is necessary for device applications. 


\section{ACKNOWLEDGEMEITS}

We thank D.G. Hinks for providing the BKBO samples, J.Z. Liu for the BSCCO samples, D.M. Ginsberg for the TBCCO samples and R.L. Greene and J.L. Peng for the NCCO samples.

\section{$\underline{\text { References }}$}

1. E.L. Wolf, Principles of Electron Tunneling Spectroscopy Ch. 2-5 (Oxford Univ. Press, New York, 1985).

2. Qiang Huang, J.F. Zasadzinski, K.E. Gray, D.R. Richards and D.G. Hinks, Appl. Phys. Lett. 57, 2356 (1990).

3. Qiang Huang, J.F. Zasadzinski, K.E. Gray, J.Z. Liu, and H. Claus, Phys. Rev. B40, 9366-9369 (1989).

4. Q. Huang et al, Nature 347, 369 (1990)

5. T. Ekino, and J. Akimitsu, Phys. Rev. B40, 7364-7367 (1989).

6. See for example, N. Miyakawa, D. Shimada, T. Kido and N. Tsuda, J. Phys. Soc. Jpn. 58, 383 (1989)

7. I. Takeuchi, J.S. Tsai, Y. Shimakawa, T. Manako and Y. Kubo, Physica C 158, 83 (1989).

8. Qiang Huang, J.F. Zasadzinski, K.E. Gray, E.D. Bukowski, and D.M. Ginsberg, Physica C161, 141-144 (1989).

9. Qiang Huar..g, J.F. Zasadzinski, and K.E. Gray Phys. Rev. B42, 7953 (1990).

10. C.K. Loong, P. Vashishta, R.K. Kalia, M.H. Degani, D.L. Price, J.D. Jorgensen, D.G. Hinks, B. Dabrowski, A.W. Mitchell, D.R. Richards and Y. Zheng, Phys.Rev. Letters 62, 2628-2631 (1989).

11. W. Reichardt et al, Proceedings of the Conference on the Manifestations of Electron-Phonon Interaction in High Temperature Superconductors, Oaxtepec, Mexico, 1990. 

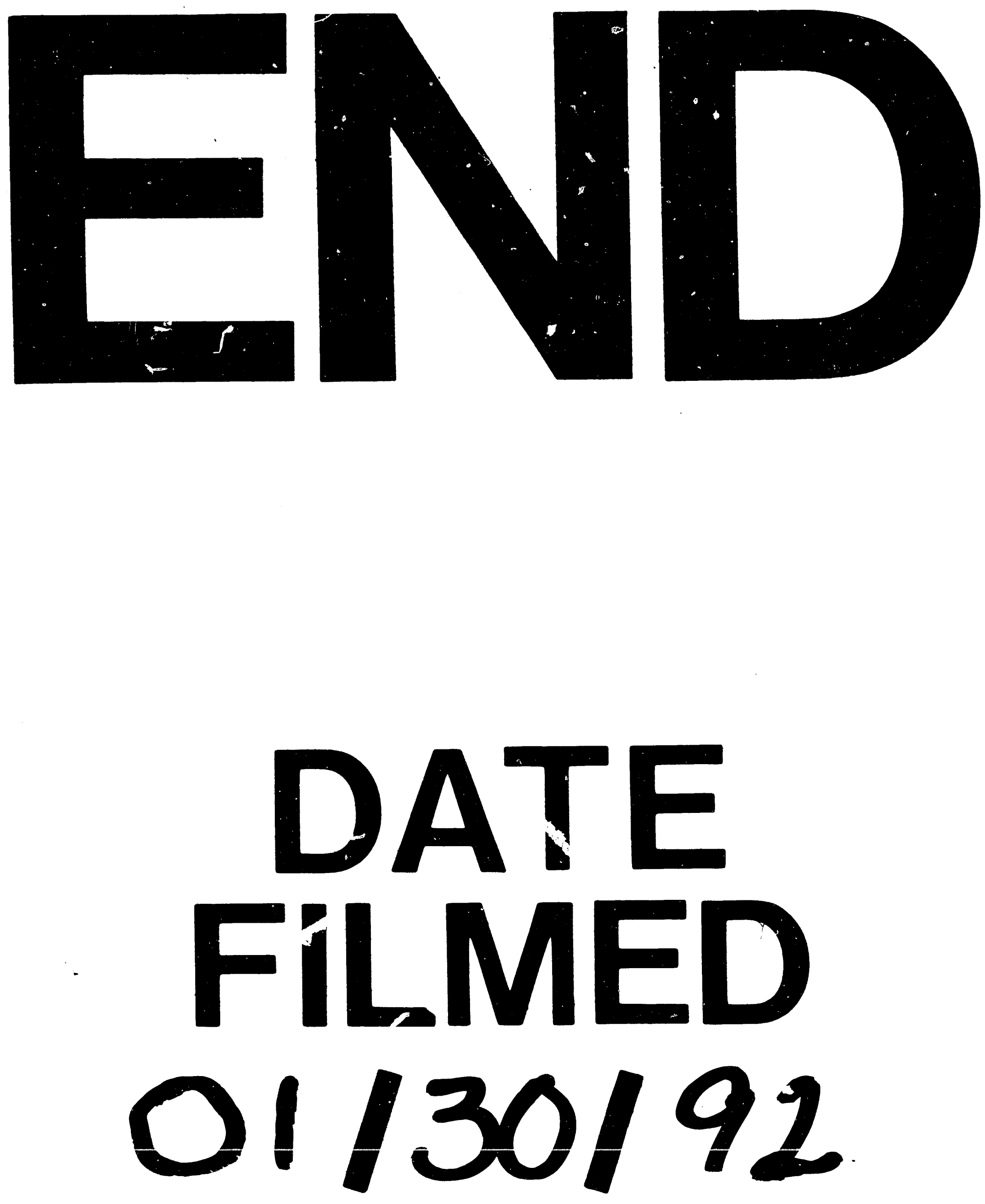

I 
$\ldots$ 